\title{
Insulin modulates energy and substrate sensing and protein catabolism induced by chronic peritonitis in skeletal muscle of neonatal pigs
}

\author{
Rodrigo Manjarín', Agus Suryawan', Sue J. Koo', Fiona A. Wilson' ', Hanh V. Nguyen', Teresa A. Davis' and Renán A. Orellana'
}

BACKGROUND: Acute infection promotes skeletal muscle wasting and insulin resistance, but the effect of insulin on energy and substrate sensing in skeletal muscle of chronically infected neonates has not been studied.

METHODS: Eighteen 2-d-old pigs underwent cecal ligation and puncture (CLP) or sham surgery (CON) to induce a chronic infection for $5 \mathrm{~d}$. On d 5, pancreatic-substrate clamps were performed to attain fasting or fed insulin levels but to maintain glucose and amino acids in the fasting range. Total fractional protein synthesis rates $(K)$, translational control mechanisms, and energy sensing and degradation signal activation were measured in longissimus dorsi muscle.

RESULTS: In fasting conditions, CLP reduced $K_{\mathrm{s}}$ and sirtuin 1 (SIRT1) and increased AMP-activated protein kinase a (AMPKa) activation and muscle RING-finger protein-1 (MuRF1). Insulin treatment increased $K_{\mathrm{s}}$ and mitochondrial protein synthesis, enhanced translation activation, and reduced SIRT1 in CON. In contrast, in CLP, insulin treatment increased $K_{s^{\prime}}$ protein kinase $\mathrm{B}$ (PKB) and Forkhead box $\mathrm{O} 1$ phosphorylation, antagonized AMPK activation, and decreased peroxisome proliferator-activated receptor gamma coactivator 1-a (PGC-1a), MuRF1, and SIRT1.

CONCLUSION: Energy and substrate sensing in skeletal muscle by the PKB-AMPK-SIRT1-PGC-1a axis is impacted by chronic infection in neonatal pigs and can be modulated by insulin.

nfection and sepsis lead to a pronounced deficit in lean body mass, with a significant proportion of the protein loss caused by the failure in maintaining normal protein synthesis rates and by the increase in protein degradation in skeletal muscle $(1,2)$. Although insulin has been associated with improving patient outcomes related to skeletal muscle dysfunction during an inflammatory response $(1,3)$, the beneficial effects of insulin on muscle catabolism are unclear. Prior studies suggest that insulin resistance for protein metabolism occurs during inflammation, but that it is affected by the severity and chronicity of the infection $(3,4)$, the age of the subject $(5-7)$, and the availability of amino acids (AAs) $(8,9)$.
In the neonatal pig, skeletal muscle protein metabolism is more sensitive and responsive to insulin stimulation than that in mature animals (10). Insulin stimulates protein synthesis in muscle by activating protein kinase $\mathrm{B}(\mathrm{PKB} / \mathrm{Akt})$, which in turn activates the mammalian target of rapamycin complex 1 (mTORC1) by inhibiting the tuberous sclerosis protein $1 / 2$ (TSC1/TSC2) complex and activating the Ras homolog enriched in brain protein (8) (Figure 1). Activated mTORC1 phosphorylates the ribosomal protein S6 kinase 1 (S6K1), which participates in protein translation initiation $(6,10,11)$. The eukaryotic initiation factor repressor 4E-binding protein 1 becomes also phosphorylated by mTORC1, releasing the eukaryotic initiation factor 4E (eIF4E) to form the active eIF4G-eIF4E complex, which binds to mRNA initiating also protein translation $(6,10,11)$. In parallel, insulin activation of PKB downregulates protein degradation by inhibiting procaspase 3 cleavage into caspase 3 , and increasing the phosphorylation of Forkhead box $\mathrm{O}$ (FOXO) transcription factors, which are excluded from the nucleus, thus decreasing the transcription of the E3 ubiquitin ligases muscle Atrophy F-Box/Atrogin-1 and muscle RINGfinger protein-1 (MuRF1) $(7,12,13)$ (Figure 1). Conversely, activation of AMP-activated protein kinase $\alpha(\mathrm{AMPK} \alpha)$ and nuclear factor- $\mathrm{\kappa B}(\mathrm{NF}-\kappa \mathrm{B})$ during energy stress and inflammation increase the expression of both ubiquitin ligases $(14,15)$. AMPK $\alpha$ activation also upregulates sirtuin 1 (SIRT1) activity, resulting in the deacetylation and activation of FOXOs (16), and increases the expression and activity of peroxisome proliferator-activated receptor gamma coactivator 1-alpha (PGC$1 \alpha$ ), a transcriptional coactivator for nuclear genes encoding mitochondrial proteins which promotes mitochondrial biogenesis and oxidative fiber switch in muscle (17).

In this study we investigated the effect of insulin treatment on muscle protein metabolism during chronic infection induced by cecal ligation and puncture (CLP) in the neonatal pig, as opposed to the acute and hypermetabolic effect of lipopolysaccharide. We hypothesize that raising insulin to fed levels ameliorates the decrease in protein synthesis and the activation of protein degradation in skeletal muscle of chronically infected neonatal pigs, and examine potential control signal interactions affected by insulin that may regulate the interface 


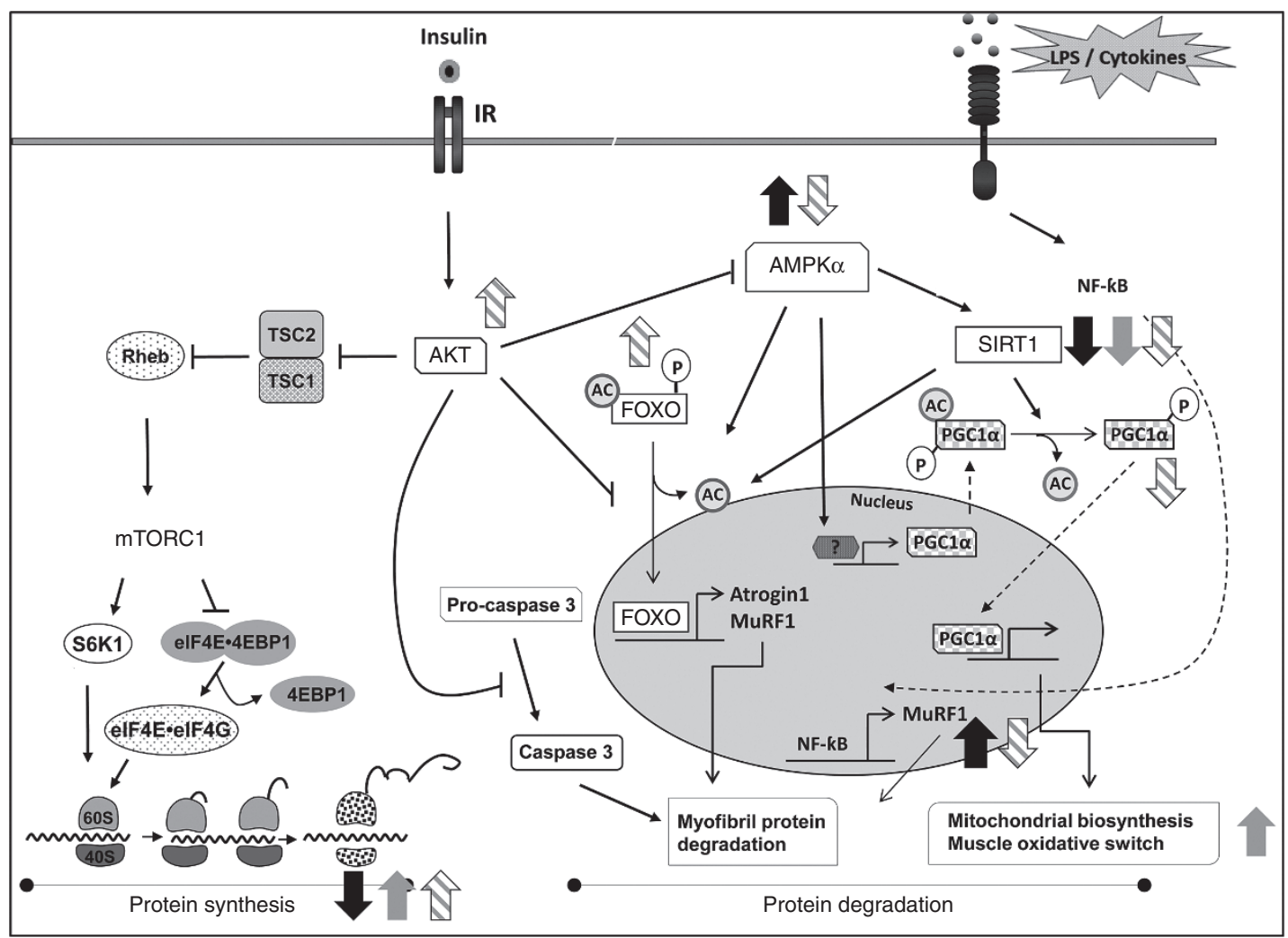

Figure 1. Schematic representation of protein synthesis, protein degradation and energy sensing signaling pathways in skeletal muscle and the effect of insulin (I) on relevant signaling after $5 \mathrm{~d}$ of cecal ligation and puncture (CLP)-induced peritonitis in neonatal pigs. $\square \mathrm{CLP} \square$ Insulin $\mathbb{Z}$ CLP + Insulin.

between substrate availability and protein turnover in neonatal skeletal muscle during chronic infection.

\section{RESULTS}

As shown in Figure 2, there were no differences in body weight gain (BWG) between CLP and control (CON) over the 5-d period. Plasma tumor necrosis factor-alpha (TNF- $\alpha$ ), interleukin-8 (IL-8), and 3-methyl histidine (3-MH) concentrations were measured on $\mathrm{d} 5$ before starting the clamp $\left(t_{0}\right)$. Concentrations of IL-8 and 3-MH in plasma were higher in CLP compared with CON ( $P=0.034$ and 0.017 , respectively), while plasma TNF- $\alpha$ levels did not differ between groups. Plasma insulin concentration was measured at $t_{0}$ to establish baseline levels, which were below $1 \mu \mathrm{U} / \mathrm{ml}$ in all groups (Table 1), and at $t_{120}$. Fasting and fed insulin levels during the clamp were targeted to be similar to those levels observed in healthy neonatal pigs (11). As given in Table 1, the concentrations of branched-chain AA (BCAA) and glucose in blood were maintained at baseline fasting levels for the duration of the clamp in the four groups. At $t_{120}$, BCAA in CLP + I were lower compared with $\mathrm{CON}+\mathrm{I}(P=0.023)$, but values remained in the fasting $\mathrm{BCAA}$ range for a neonatal pig. As shown in Figure 3, whole body glucose and AA disposal rates were calculated from the infusion rates of dextrose and AA solution needed to maintain their baseline levels during the clamp. Compared with CON, CLP did not affect glucose and AA disposal rates, whereas insulin increased glucose disposal rates in CLP $(P=0.028)$ and AA disposal rates in both CON and CLP groups ( $P=0.005$ and 0.014 , respectively).

In Figure 4, CLP decreased the total fractional protein synthesis rate $\left(K_{\mathrm{s}}\right)$ in longissimus dorsi compared with $\mathrm{CON}(P=$ $0.009)$. Insulin increased total $K_{\mathrm{s}}$ in longissimus dorsi in both CON and CLP groups ( $P=0.015$ and 0.0005 , respectively), and it was higher in CON + I compared with CLP + I $(P=0.008)$. Mitochondrial fractional protein synthesis rate in longissimus dorsi was unaffected by CLP $(P=0.56)$, and increased only in CON in response to insulin $(P=0.044)$.

In Figure 5 is shown the treatment effect on the activation of several mTOR pathway substrates involved in protein translation initiation. Compared with CON, CLP did not affect phosphorylation of PKB $(P=0.84)$, TSC2 $(P=0.80)$, mTOR $(P=0.89)$, S6K1 $(P=0.84)$, or eIF4E-eIF4G $(P=0.78)$. In $\mathrm{CON}$, insulin increased the phosphorylation/abundance of all measured proteins $(P=0.0003,0.007,0.004,0.013$, and 0.002 for PKB, TSC2, mTOR, S6K1, and eIF4E-eIF4G, respectively), whereas only PKB $(P=0.007)$, TSC2 $(P=0.038)$, and mTOR $(P=0.004)$ were increased in CLP in response to insulin. Phosphorylation of mTOR was higher in CLP + I compared with $\mathrm{CON}+\mathrm{I}(P=0.032)$.

In Figures 6 and 7 is shown the treatment effect on the activation of several factors involved in muscle protein degradation. CLP decreased phosphorylation of FOXO3 $(P=0.048)$ and increased the abundance of MuRF1 compared with CON $(P=0.008)$, whereas insulin increased phosphorylation of 

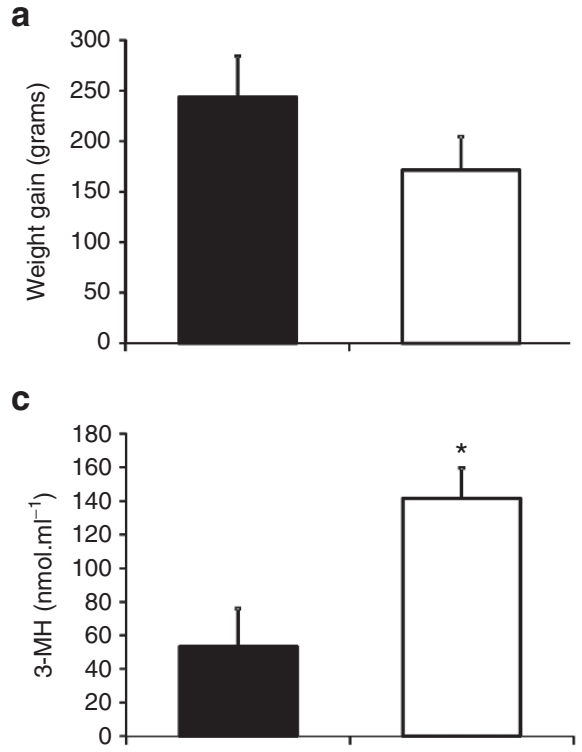

b

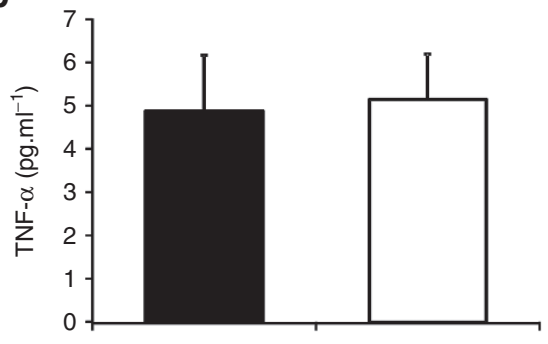

d

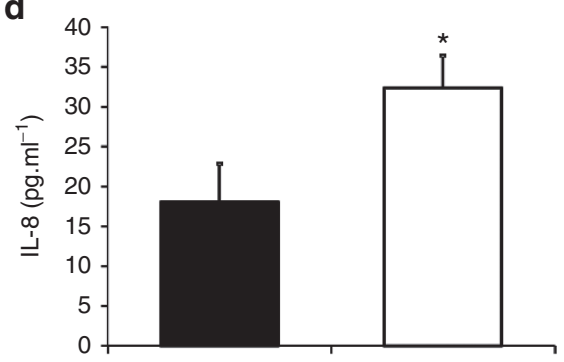

Figure 2. Body weight gain (a) and plasma concentrations of tumor necrosis a (TNF-a)(b), interleukin 8 (IL-8) (d), and 3-methylhistidine (3-MH)(c), after $5 \mathrm{~d}$ of CLP-induced peritonitis in neonatal pigs. Black bars represent sham animals while white bars represent CLP animals. Values are expressed as least square means $\pm \mathrm{SE}$. CON, control $(n=9) ; \mathrm{CLP}$, cecal ligation and puncture $(n=9){ }^{*} P=0.05$.

Table 1. Plasma concentration of insulin $(\mu \mathrm{U} / \mathrm{ml})$, branched-chain amino acids $(\mathrm{nmol} / \mathrm{ml})$, and glucose $(\mathrm{mg} / \mathrm{dl})$ in neonatal pigs during a 2-h pancreatic-substrate clamp after $5 \mathrm{~d}$ of peritonitis

\begin{tabular}{lcc}
\hline Item & Baseline $\left(t_{0}\right)$ & Clamp $\left(t_{120}\right)$ \\
\hline CON & & \\
Insulin & $0.5 \pm 2.0$ & $5.6^{*} \pm 2.0$ \\
BCAA & $613.8 \pm 83.4$ & $463.1 \pm 83.4$ \\
Glucose & $45.0 \pm 6.0$ & $51.0 \pm 6.0$ \\
CON + Insulin & & \\
Insulin & $0.2 \pm 2.0$ & $29.2^{*} \pm 2.0$ \\
BCAA & $759.0 \pm 83.4$ & $596.1 \pm 83.4$ \\
Glucose & $42.1 \pm 6.0$ & $56.6 \pm 6.0$ \\
CLP & & \\
Insulin & $0.5 \pm 2.0$ & $4.9^{*} \pm 2.0$ \\
BCAA & $563.5 \pm 83.4$ & $492.0 \pm 83.4$ \\
Glucose & $46.2 \pm 6.1$ & $59.6 \pm 6.1$ \\
CLP + Insulin & & $32.7^{*} \pm 1.8$ \\
Insulin & $0.4 \pm 1.8$ & $389.5^{* *} \pm 74.6$ \\
BCAA & $444.5 \pm 74.6$ & $55.0 \pm 5.4$ \\
Glucose & $50.2 \pm 5.4$ &
\end{tabular}

Five days after cecal ligation and puncture (CLP), insulin was targeted to fasting levels $(2-5 \mu \mathrm{U} / \mathrm{ml})$ or fed levels $(\sim 30 \mu \mathrm{U} / \mathrm{ml})$ while amino acids and glucose were maintained at baseline fasting levels. Values are expressed as means $\pm \mathrm{SE}$.

BCAA, branched-chain amino acids; CON, control, $n=4 ; \mathrm{CON}+$ Insulin, $n=5 ; \mathrm{CLP}, n=4$; CLP + Insulin, $n=5$.

*Significantly different between baseline and clamp levels $(P<0.001)$. * Significantly different between groups $(P<0.05)$.

FOXO1 and Pro-caspase 3 abundance $(P=0.002$ and 0.034 , respectively) and decreased MuRFlabundance $(P=0.003)$ in CLP. Phosphorylation of FOXO4 and atrogin-1 abundance were unaffected by CLP ( $P=0.56$ and 0.34 , respectively) or insulin $(P=0.69$ and 0.72 , respectively). Phosphorylation of NF- $\kappa B$ increased in CLP compared with CON $(P=0.014)$ but was unaffected by insulin. Compared with CON, CLP increased phosphorylation of AMPK $\alpha(P=0.02)$ and decreased SIRT1 abundance $(P=0.0004)$. Insulin decreased the phosphorylation of AMPK $\alpha$ and SIRT1 abundance in CLP $(P=0.002$ and 0.0008 , respectively), and SIRT1 alone in CON $(P=0.0002)$. Both AMPK $\alpha$ and SIRT1 were lower in CLP + I compared with CON + I $(P=0.009$ and $<0.0001$, respectively). mRNA abundance of PGC1- $\alpha$ was unaffected by CLP and decreased in response to insulin in CLP $(P=0.03)$.

\section{DISCUSSION}

In this study, we assessed the effect of insulin treatment on muscle protein metabolism using a long-term $(5 \mathrm{~d})$ survival model of peritonitis in neonatal pigs, which may resemble the effects of chronic infection $(2,18)$. Of note, in newborn piglets, skeletal muscle protein synthesis is extremely sensitive to insulin stimulation when compared with more mature swine. By performing a pancreatic substrate clamp to manipulate insulin and maintain glucose and AA at fasting levels in the presence of a chronic abdominal infection, we intend to determine in vivo possible signaling restraints that may affect the insulin effect on muscle protein homeostasis. However, in a clinical scenario, many variables modify the intensity and effect of insulin in skeletal muscle, including age, body mass, developmental physiology, the type and duration of the inflammatory insult, and therefore may limit the accuracy of our findings.

CLP increased plasma levels of IL-8 and 3-MH, a component of the myofibrils released during skeletal muscle degradation (19), suggesting an ongoing inflammatory and protein catabolic process similar to that observed in CLP rodent models (4). Interestingly, the increase in IL-8 and 3-MH levels was 
a

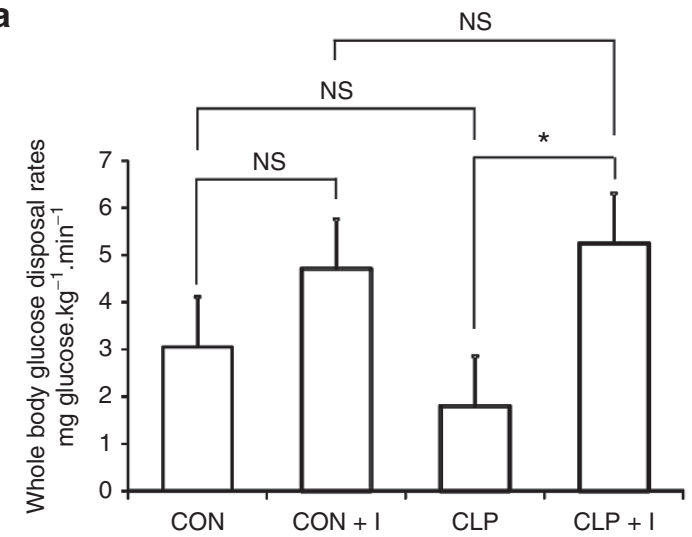

b

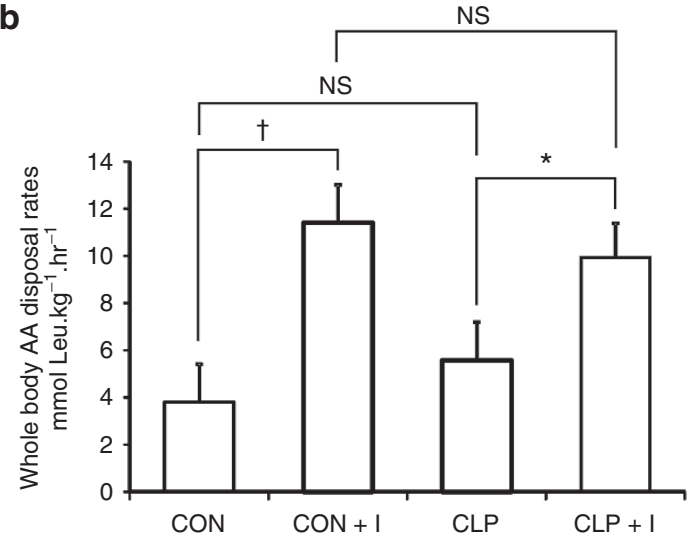

Figure 3. Net whole body glucose (a) and amino acid (AA) disposal rates (b) during a 2-h pancreatic glucose-AA clamp after $5 \mathrm{~d}$ of peritonitis in neonatal pigs. CON, control, $n=4 ; \mathrm{CON}+$ Insulin, $n=5 ; \mathrm{CLP}$, cecal ligation and puncture, $n=4 ; \mathrm{CLP}+\operatorname{Insulin}, n=5$. Values are means $\pm \mathrm{SE} ; n=4-5$ per group. ${ }^{*} P=0.05,{ }^{* *} P=0.001$.
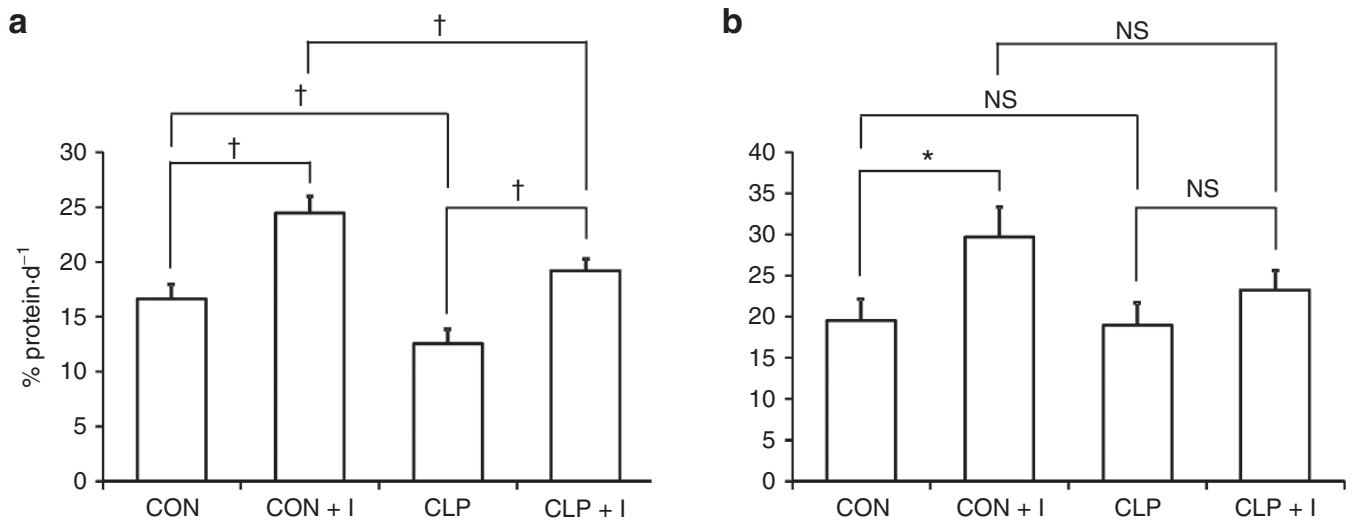

Figure 4. Global (a) and mitochondrial (b) fractional protein synthesis rates during a 2-h pancreatic glucose-amino acid clamp after $5 \mathrm{~d}$ of peritonitis in skeletal muscle of neonatal pigs. CON, control, $n=4 ; \mathrm{CON}+$ Insulin, $n=5 ; \mathrm{CLP}$, cecal ligation and puncture, $n=4$; CLP + Insulin, $n=5 .{ }^{*} P=0.05,{ }^{* *} P=0.001$.

not paired to higher TNF- $\alpha$ concentrations in blood. Studies in mice have shown changes in plasma cytokine levels to be related to needle size and location of the cecal ligature $(4,18)$. Since we performed small cecal punctions to allow a 5-d survival, the insult may not have been severe enough to maintain elevated TNF- $\alpha$ plasma levels throughout the study. Similarly, low TNF- $\alpha$ concentrations have been also observed in surviving or non-bacteremic patients and in long-term murine survivors of CLP $(20,21)$. In addition, in newborns, IL-8, rather than TNF- $\alpha$, has been shown to be a more important mediator in the pathogenesis of neonatal sepsis (22). BCAA levels were lower in CLP + I compared with CON + I, indicating higher whole-body AA requirements in response to the inflammatory process (5). These results suggest that we developed a low-intensity chronic infectious model with ongoing muscle catabolism.

We have previously shown that baseline muscle protein synthesis rates $\left(K_{\mathrm{s}}\right)$ and translation activation were reduced in endotoxemic piglets, even in the presence of insulin (5). Likewise, in this study $K_{\mathrm{s}}$ was lower in CLP compared with $\mathrm{CON}$ regardless of despite adequate insulin stimulation and AA deposition, whereas insulin failed to activate S6K1 and eIF4EeIF4G in CLP animals despite the presence of an increase in muscle protein synthesis in response to insulin stimulation. These results are in agreement with previous studies $(1,2,5)$ and suggest that during an acute and chronic infection there is a translational defect downstream of mTOR which attenuates muscle protein synthesis in response to insulin. Moreover, our group and several others have shown that in animals and humans in a variety of conditions, the activation of an intracellular signaling cascade does not necessarily correlate with the expected physiological effect, especially when the molecular analysis is performed in vivo.

Mitochondrial protein synthesis is a highly complex and poorly understood determinant of metabolic adaptation (23). In noninflammatory conditions, mitochondrial translation is coregulated with cytoplasmic translation, and thus during starvation and normal conditions, protein synthesis in both the cytoplasm and within the mitochondria plays a role in this adaptive response by modulating their ATP generating capacity through synthesis of oxidative phosphorylation enzymes (24). Therefore, mTOR and mitochondrial translation act as components of the major nutrient sensing apparatus integrating nutrient supply into the control of protein homeostasis $(23,24)$. In normal conditions, mitochondrial protein synthesis has been shown to respond to insulin stimulation (8). In our study, 


\section{Articles | Manjarín et al.}

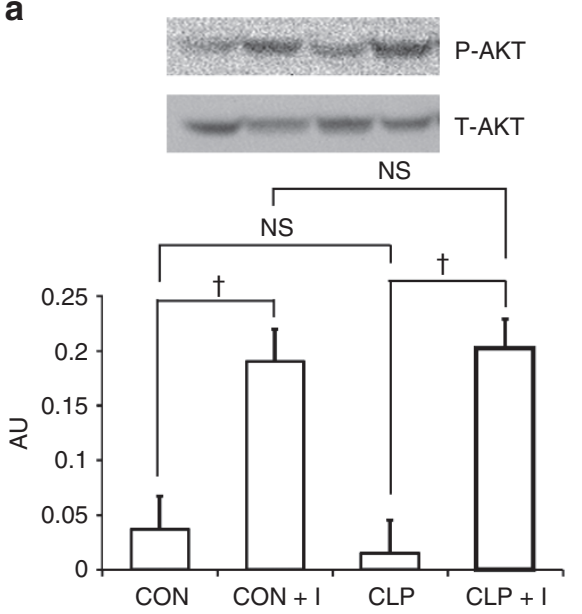

b

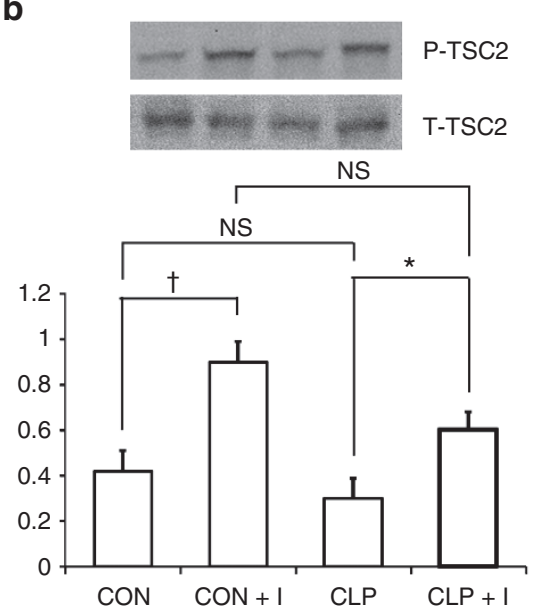

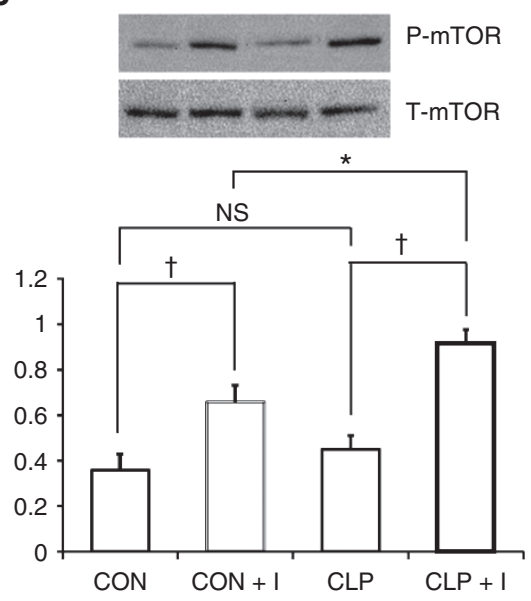

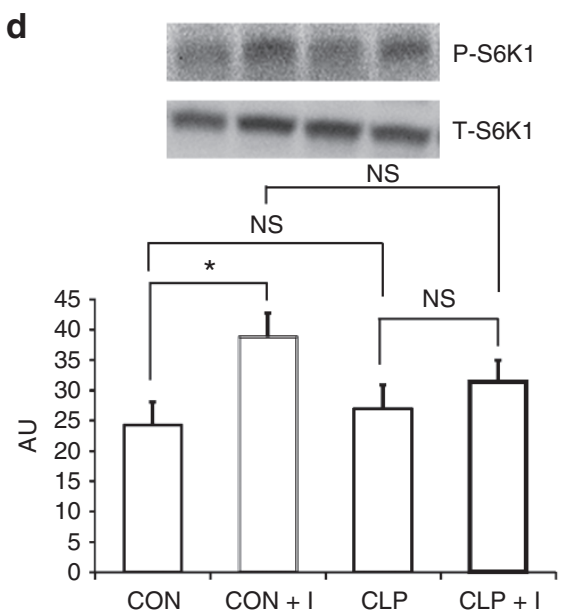

e

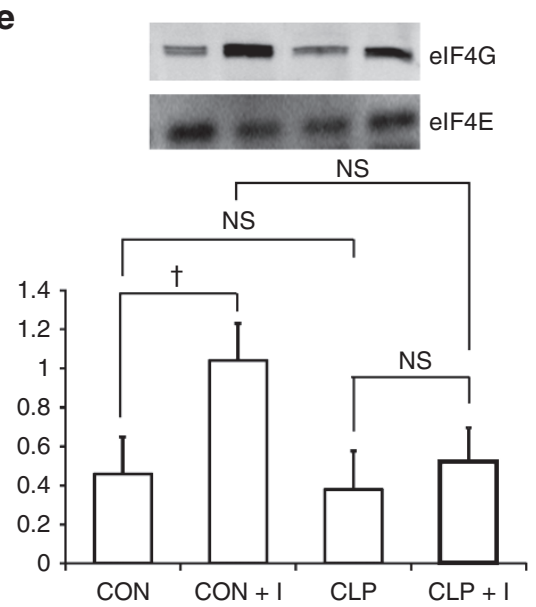

Figure 5. Effect of insulin on the phosphorylation of protein kinase B (p-PKB) (a), tuberous sclerosis complex 2 (p-TSC2) (b), mammalian target of rapamycin (p-mTOR) (c), ribosomal protein 56 kinase-1 (p-S6K1) (d), and the abundance of eukaryotic initiation factor 4 complex (elF4E-elF4G) (e) during a 2-h pancreatic glucose-amino acid clamp after $5 \mathrm{~d}$ of peritonitis in skeletal muscle of neonatal pigs. CON, control, $n=4 ; \mathrm{CON}+$ Insulin, $n=5 ; \mathrm{CLP}, \mathrm{cecal}$ ligation and puncture, $n=4 ; \mathrm{CLP}+$ Insulin, $n=5$. Values are means $\pm \mathrm{SE}$. ${ }^{*} P=0.05,{ }^{*} P=0.001$.

mitochondrial protein synthesis in sham animals increased in response to insulin stimulation, but this response was abrogated by CLP. Since rodents subjected to 5 -d peritonitis as a septic insult have shown changes in global protein synthesis rates in skeletal muscle, but no changes in intracellular ATP (2), it is possible that the lack of response of mitochondrial protein synthesis to insulin in CLP animals is not related to impairment of synthesis of oxidative phosphorylation enzymes and energy generation, but to a decrease in the adaptive capacity to stress through biogenesis, as described below.

Protein abundance of MuRF1 was increased in CLP animals and downregulated by insulin; however, it did not correlate with $\mathrm{PKB}$ and FOXO1 phosphorylation in $\mathrm{CON}$, suggesting that MuRF1 regulation by $\mathrm{PKB}-\mathrm{FOXO1}$ axis may occur only during inflammation. Given that NF- $\kappa \mathrm{B}$ is mainly activated by TNF- $\alpha(7,25)$ and CLP failed to increase plasma TNF- $\alpha$ levels, it is possible that upregulation of NF- $\kappa B$ in CLP animals was not of sufficient magnitude for insulin to decrease it.

In skeletal muscle, AMPK $\alpha$ functions as a fuel sensor and its phosphorylation decreases muscle protein synthesis, enhances proteolysis by increasing atrogin-1 and MuRF1, and can be antagonized by insulin through PKB activation $(7,26)$. In agreement with these studies, insulin antagonized the increase in AMPK $\alpha$ phosphorylation induced by CLP, in parallel with a decrease in MuRF1. AMPK also upregulates the expression and activity of PGC-1 $\alpha$ (Figure 1) $(27,28)$, which promotes mitochondrial biogenesis and oxidative switch (17). Accordingly, we found PGC- $1 \alpha$ mRNA expression to correlate with the insulin-induced decrease in AMPK $\alpha$ phosphorylation in the CLP group, suggesting an improvement on energy balance in muscle in response to insulin.

Activation of AMPK $\alpha$ also leads to the upregulation of SIRT1 activity, a metabolic sensor that has been shown to impair metabolic adaptation and myogenesis in skeletal muscle cells (16). Data from our study indicate for the first time a decrease in SIRT1 in response to insulin in both CLP and CON. Given that SIRT1 expression and function have been positively correlated in mouse skeletal muscle (29), it is possible that SIRT1 downregulation represents another mechanism of control by which insulin attenuates muscle protein degradation in septic neonates. 
a

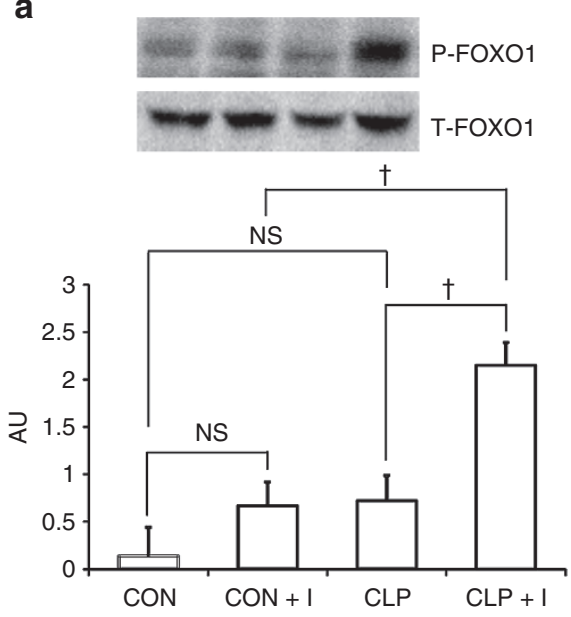

d

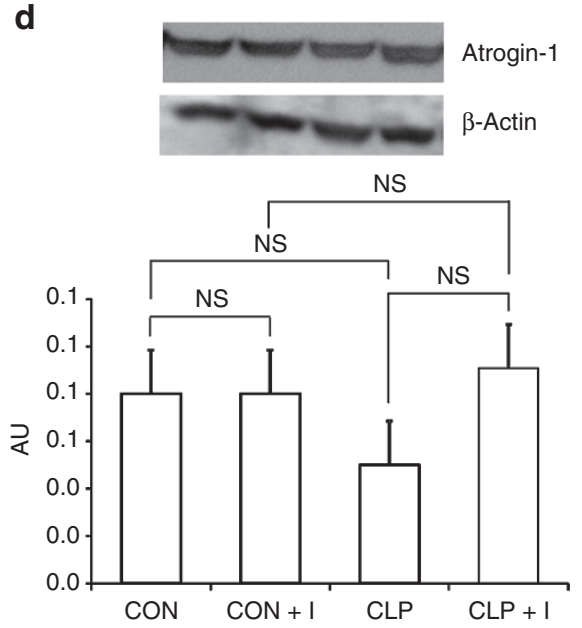

b

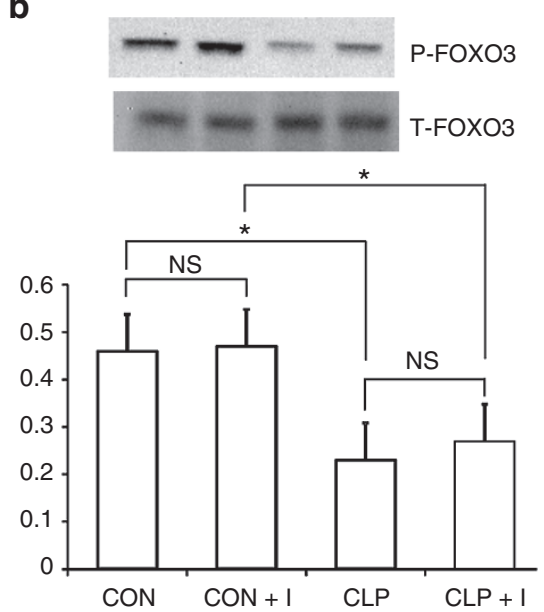

e

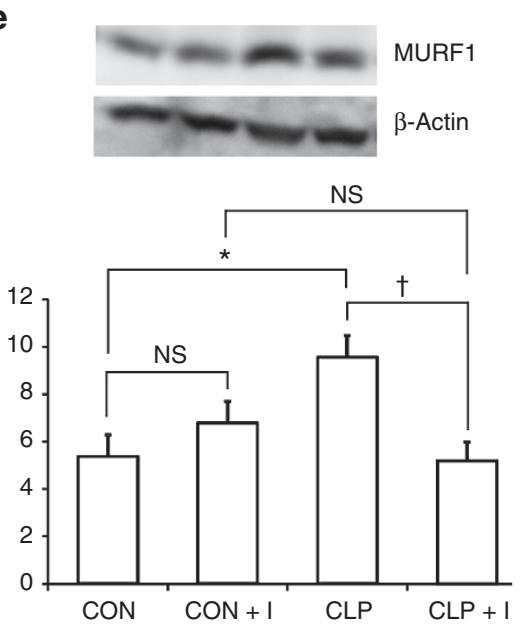

c

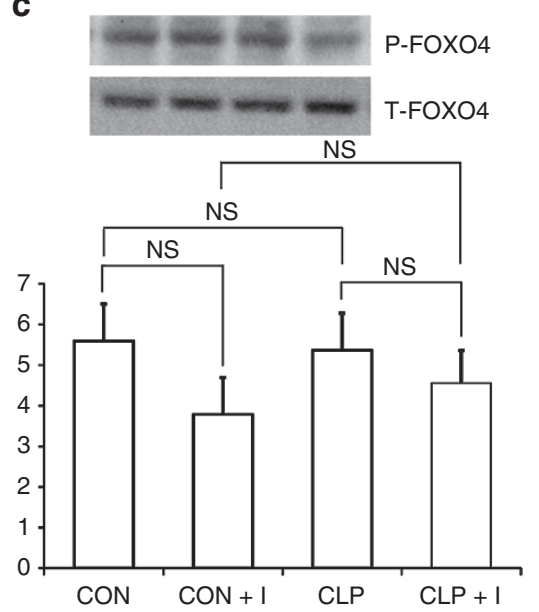

f
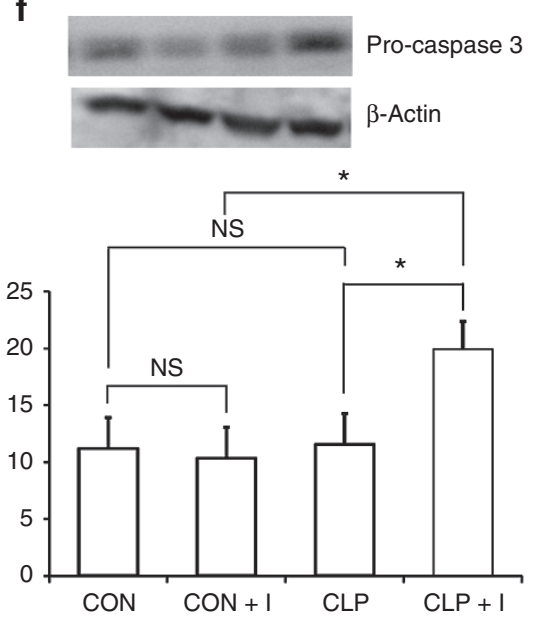

Figure 6. Effect of insulin on the phosphorylation of forkhead box $\mathrm{O} 1$ (FOXO1) (a), FOXO3 (b), and FOXO4 (c), and the abundance of atrogin-1/Muscle Atrophy F-box (Atrogin-1) (d), muscle RINGfinger protein-1 (MuRF1) (e), and Pro-caspase 3 (f) during a 2-h pancreatic glucose-amino acid clamp after $5 \mathrm{~d}$ of peritonitis in skeletal muscle of neonatal pigs. CON, control, $n=4 ; \mathrm{CON}+$ Insulin, $n=5 ; \mathrm{CLP}$, cecal ligation and puncture, $n=4 ; \mathrm{CLP}+\operatorname{Insulin}, n=5$. Values are means $\pm \mathrm{SE} .{ }^{*} P=0.05,{ }^{* *} P=0.001$.

In conclusion, our results suggest that insulin targets the AMPK-FOXO-SIRT1-PGC-1 $\alpha$ axis, interferes with energy and substrate sensing, and antagonizes the catabolic effect of chronic infection in skeletal muscle. Future studies will examine whether AMPK-FOXO-SIRT1-PGC-1 $\alpha$ axis is regulated during neonatal growth, and whether is impacted by dietary AAs, and specifically leucine.

\section{Perspective and Significance}

Anabolic failure contributes to the inefficiency of feeding regimens during chronic infections, resulting in loss of lean body mass and poor recovery. Understanding the interface between insulin stimulation, substrates, and the PKB-FOXO-MuRF1 and AMPK $\alpha-$ SIRT1-PGC1 $\alpha$ axes as shown in Figure 1 may improve the outcome of skeletal muscle wasting in patients recovering from infection.

\section{METHODS}

\section{Animals and Experimental Design}

The experimental design was approved by the Animal Care and Use Committee of Baylor College of Medicine (Figure 8). Eighteen 1-d-old crossbred piglets were weaned in two consecutive replicates ( $n=10$ and 8), housed in individual cages, and fed ad libitum a commercial diet. At $2 \mathrm{~d}$ of age $(2.02 \pm 0.12 \mathrm{~kg})$ jugular and carotid catheters were placed and flushed with heparinized saline $(100 \mathrm{IU} / \mathrm{ml}$; APP, Lake Zurich, IL) every $48 \mathrm{~h}$. Ten pigs underwent ligation and double perforation of the cecum (CLP) using an 18-gauge needle and manual expression of the cecum fecal content, while the remaining eight pigs underwent sham laparotomy to expose the cecum and were used as controls (CON). Piglets were pair-fed by gavage during $5 \mathrm{~d}$ $40 \mathrm{ml} / \mathrm{kg} \mathrm{BW}$ at 6-h intervals. Surgery day will be considered as d 0 for the remainder of the study. On d 5, all animals were killed with an injection of pentobarbital sodium $(0.4 \mathrm{ml} / \mathrm{kg} \mathrm{BW})$. Tissue samples were obtained from the longissimus dorsi, flash frozen in liquid $\mathrm{N}$, and stored at $-80^{\circ} \mathrm{C}$.

\section{Pancreatic Glucose-AA Clamps}

On d 5, after overnight fasting, animals were placed in a sling restraint system to perform pancreatic-substrate clamps for $2 \mathrm{~h}$, as previously described $(5,11)$. The clamp was initiated by infusing $100 \mu \mathrm{g} / \mathrm{kg} / \mathrm{h}$ of somatostatin (Bachem, Torrance, CA). Glucose and AAs were also clamped \pm 10 th percentile of the individual baseline fasting levels by monitoring their concentration every $5 \mathrm{~min}$ and adjusting the infusion rate of $10 \%$ dextrose $(\sim 60-80 \mathrm{mg} / \mathrm{dl})$ and a balanced mixture of essential and nonessential AAs $(\sim 500 \mathrm{nmol} \mathrm{BCAA} / \mathrm{ml})(5,11)$.

Fifteen min after starting the somatostatin infusion, replacement glucagon (150 ng/kg/h; Sigma-Aldrich, Milwaukee, WI) and insulin 
a

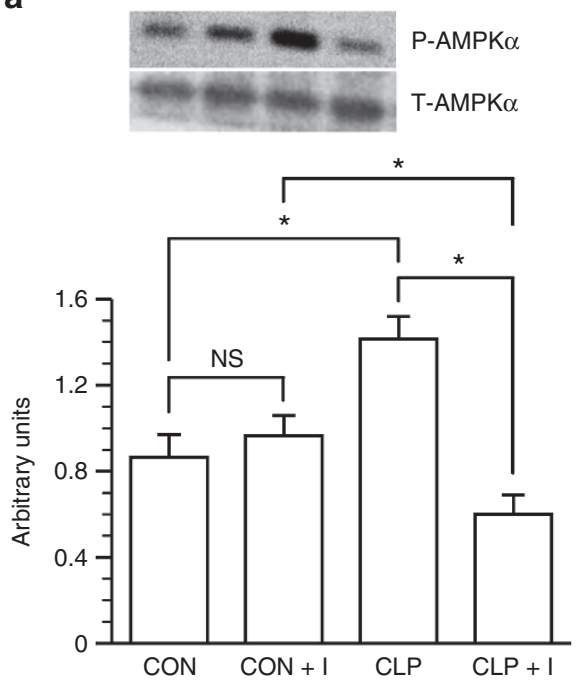

C

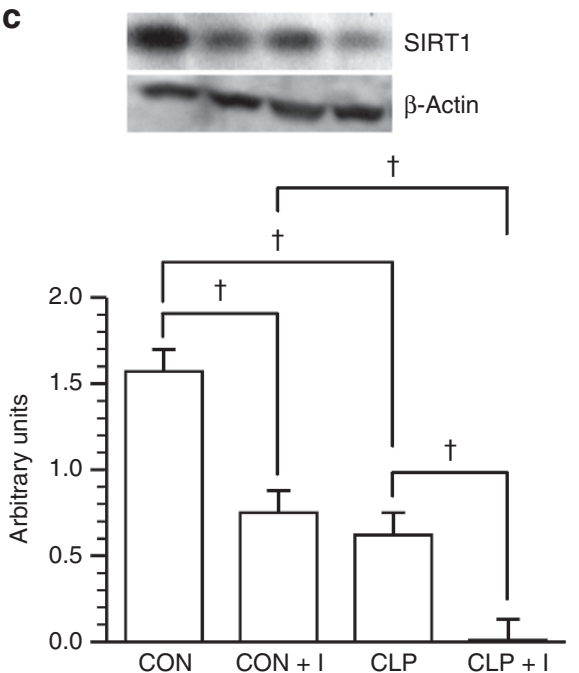

b
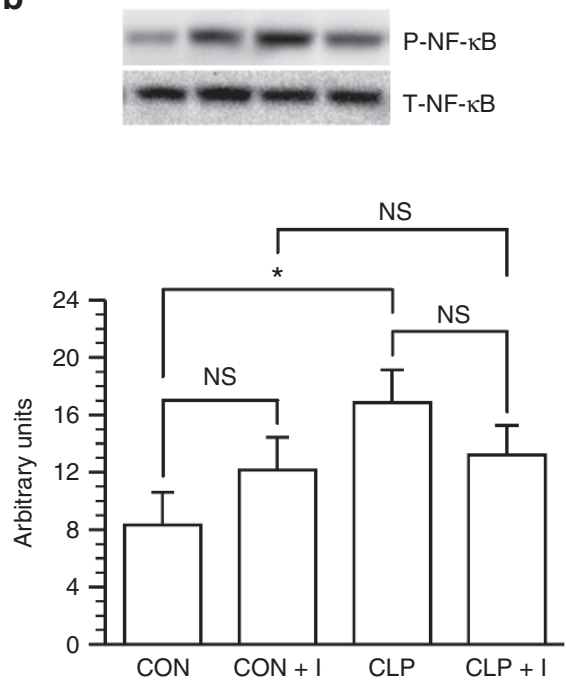

d

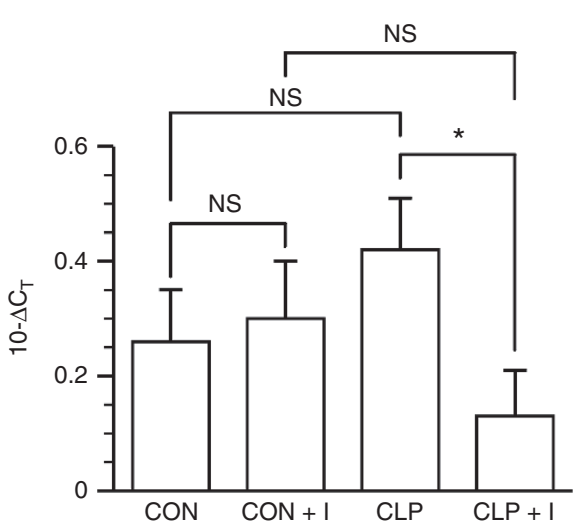

Figure 7. Effect of insulin on the phosphorylation of AMP-activated protein kinase $\alpha$ (p-AMPK $\alpha)(\mathbf{a})$, and nuclear factor-KB (NF-kb) (b), protein abundance of sirtuin 1 (SIRT1) (c), and the mRNA abundance of peroxisome proliferator-activated receptor gamma coactivator1 a (PGC-1a) (d) during a 2-h pancreatic glucose-amino acid clamp after $5 \mathrm{~d}$ of peritonitis in skeletal muscle of neonatal pigs. CON, control, $n=4 ; \operatorname{CON}+\operatorname{Insulin}, n=5 ; \mathrm{CLP}, \mathrm{cecal}$ ligation and puncture, $n=4 ; \mathrm{CLP}+$ Insulin, $n=5$. Values are means $\pm \mathrm{SE}$. ${ }^{*} P=0.05,{ }^{* *} P=0.001$.

at either $7 \mathrm{ng} / \mathrm{kg}^{0.66} / \mathrm{min}$ to achieve plasma concentrations of $2-5 \mu \mathrm{U} /$ $\mathrm{ml}$ to simulate a fasting insulinemic state (CON, $n=4$; CLP, $n=4)$, or at $40 \mathrm{ng} / \mathrm{kg}^{0.66} / \mathrm{min}(\mathrm{CON}+\mathrm{I}, n=5 ; \mathrm{CLP}+\mathrm{I}, n=5)$ to replicate fed levels of insulin $(\sim 30 \mu \mathrm{U} / \mathrm{ml})$ observed in healthy neonatal pigs, were also infused.

\section{Circulating Insulin, Cytokines, and Substrate Measurements}

On d 5, blood samples were taken before starting the pancreatic glucose-AA clamp $\left(t_{0}\right)$ and at $120 \mathrm{~min}\left(t_{120}\right)$. Samples were centrifuged at $12,000 \mathrm{~g}$ for $2 \mathrm{~min}$ within $10 \mathrm{~min}$ of collection, and plasma was stored at $-20^{\circ} \mathrm{C}$. Plasma insulin concentrations were measured using porcine insulin radioimmunoassay kits (Linco, St. Charles, $\mathrm{MO}$ ). Determination of 3-MH was performed by high-performance liquid chromatography (PICO-TAG reverse-phase column; Waters, Mildford, MA). Plasma concentrations of TNF- $\alpha$ and IL- 8 were measured using solid-phase sandwich enzyme-linked immunosorbent assays with swine antibodies against the respective cytokines and standards (Biosource International, Camarillo, CA).

\section{Global and Mitochondrial Protein Synthesis Measurements}

Protein synthesis was measured as previously described $(5,10,11)$. For mitochondrial synthesis measurements, isolation of subsarcolemmal mitochondria was performed using a method described previously (30). Briefly, muscle was homogenized with buffer A ( $0.25 \mathrm{M}$ sucrose, $2 \mathrm{mM}$ ethylenediaminetetraacetate, and $10 \mathrm{mM}$ Tris- $\mathrm{HCl} ; \mathrm{pH} 7.4$ ), centrifuged at $10,000 \mathrm{~g}$ for $10 \mathrm{~min}$ at $4^{\circ} \mathrm{C}$, and the resulting supernatant was recentrifuged at $10,000 \mathrm{~g}$ for $15 \mathrm{~min}$ at $4^{\circ} \mathrm{C}$. The resultant precipitate was then dissolved with buffer B $(100 \mathrm{mM} \mathrm{KCl}, 5 \mathrm{mM}$ ethyleneglycol-bis(baminoethylether)- N,N9-tetraacetic acid, $5 \mathrm{mM} \mathrm{MgSO}_{4}$, $1 \mathrm{mM}$ ATP, and $50 \mathrm{mM}$ Tris-HCl; $\mathrm{pH}$ 7.4) and recentrifuged at 10,000g at $4^{\circ} \mathrm{C}$ twice. The resultant precipitate was dissolved in buffer $\mathrm{A}$ and a fraction was used to detect mitochondrial oxidative phosphorylation proteins by western blotting (figure not shown). If mitochondrialspecific enzymes were detected, the rest of the prep was centrifuged at $10,000 \mathrm{~g}$ for $5 \mathrm{~min}$ and subjected to analysis of the specific radioactivity of tissue-free L-[4-3H]-Phe, as described previously $(5,11)$.

Western blot analyses were performed using methods described previously (6). Primary antibodies used in the immunoblotting were against PKB/Akt (total and Ser473; Cell Signaling Technology, Beverly, MA), TSC2 (total and Thr1462; Cell Signaling), mTOR (total and Ser2448; Cell Signaling), S6K1 (total and Thr389; Cell Signaling), AMPKo (total and Thr172; Cell Signaling), atrogin-1 (ECM Biosciences, Versailles, KY), MuRF1 (R\&D Systems, Minneapolis, MN), FOXO1 (total and 


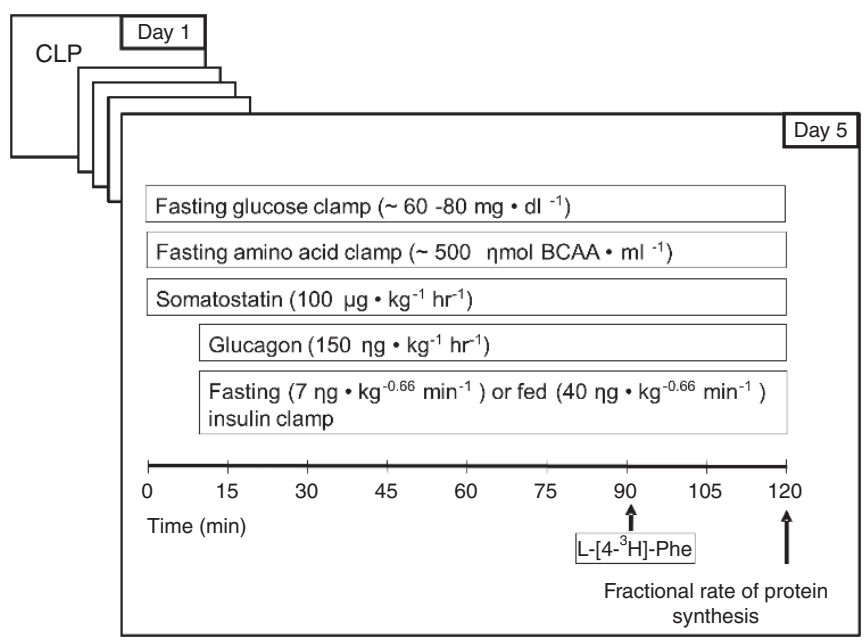

Figure 8. Schematic representation of the pancreatic glucose-AA clamp after $5 \mathrm{~d}$ of cecal ligation and puncture (CLP) in neonatal pigs. AA, amino acid; Phe, phenylalanine.

Ser 256; Cell Signaling), FOXO3 (total and Ser253; Cell Signaling), FOXO4 (total and Ser262; Cell Signaling), NF- $\mathrm{BB}$ (total and Ser536; Cell Signaling), SIRT1 (Cell Signaling), Pro-caspase 3 (Cell Signaling), and eIF4E-eIF4G (gift of Dr Leonard Jefferson, College of Medicine, Hershey, PA).

\section{PGC-1 $\alpha$ Expression}

Ribonucleic acid was extracted from tissue using Qiazol (Qiagen, Valencia, CA) and tested for quality using an Agilent Bioanalyzer 2100 with the RNA 6000 Nano Labchip (Agilent Technologies, Palo Alto, CA). Total RNA was treated with DNase I (Roche, Indianapolis, IN) and reverse-transcribed using High Capacity RNA-cDNA Master Mix (Applied Biosystems, Foster City, CA). Primer sequences for PGC$1 \alpha$ (GenBank\#NM_213963.1; 5' -CCTGCATGAGTGTGTGCTCT-3' and $5^{\prime}$-CTCAGAGTCCTGGTTGCACA-3') and 18S ribosomal RNA (GenBank\#NR_002170; 5'-AGGGCATCACAGACCTGTTATTG-3' and $5^{\prime}$-CCCCAACTTCTTAGAGGGACAG-3') were designed using Primer Express software (v. 3.0; Applied Biosystems). Samples were analyzed in duplicate in MicroAmp Optical 96-Well Reaction Plates (Applied Biosystems), using $30 \mathrm{ng}$ of cDNA, $12.5 \mu \mathrm{l}$ Fast SYBR Green Master Mix (Applied Biosystems), and $200 \mathrm{nM}$ of each primer. The amplification program consisted on $95^{\circ} \mathrm{C}$ for $10 \mathrm{~min}, 40$ cycles of $95^{\circ} \mathrm{C}$ for $15 \mathrm{~s}$ and $60^{\circ} \mathrm{C}$ for $1 \mathrm{~min}$, and a final dissociation curve (ABI 7500 Fast Sequence Detection System; Applied Biosystems). Data were analyzed with the 7500 RQ Sequence Detection Systems Software (v. 2.2.1; Applied Biosystems). Results are presented as target gene fold changes, according to the $2^{-\Delta \Delta C t}$ method (31).

\section{Statistical Analyses}

Data analysis was performed by a two-way ANOVA and Pearson correlation on SAS 9.2 (SAS Institute Inc., Cary, NC). $P$-values for preplanned pairwise comparisons were calculated using $t$-tests. Data are presented as least square means $\pm \mathrm{SE}$. Significant effects were considered at $P<0.05$.

\section{ACKNOWLEDGMENTS}

We thank Jerome Stubblefield for assistance with care of animals and Rosemarie Almonaci for technical support.

\section{STATEMENT OF FINANCIAL SUPPORT}

The authors do not have any financial ties to products in the study. This project has been funded in part by the National Institutes of Health awards, K08AR-51563 (R.A.O.), National Institute of Child Health and Human Development HD-072891 (T.A.D.) and R01 AR-44474 (T.A.D.), and the US Department of Agriculture, Agricultural Research Service under Cooperative Agreement number 6250-51000-055 (T.A.D.).

Disclosure: The authors do not have any conflicts of interest to disclose.

\section{REFERENCES}

1. Lang CH, Frost RA, Vary TC. Regulation of muscle protein synthesis during sepsis and inflammation. Am J Physiol Endocrinol Metab 2007;293:E453-9.

2. Vary TC, Siegel JH, Tall BD, Morris JG, Smith JA. Inhibition of skeletal muscle protein synthesis in septic intra-abdominal abscess. J Trauma 1988;28:981-8.

3. Whyte MB, Jackson NC, Shojaee-Moradie F, et al. Metabolic effects of intensive insulin therapy in critically ill patients. Am J Physiol Endocrinol Metab 2010;298:E697-705.

4. Ebong S, Call D, Nemzek J, Bolgos G, Newcomb D, Remick D. Immunopathologic alterations in murine models of sepsis of increasing severity. Infect Immun 1999;67:6603-10.

5. Orellana RA, Kimball SR, Suryawan A, et al. Insulin stimulates muscle protein synthesis in neonates during endotoxemia despite repression of translation initiation. Am J Physiol Endocrinol Metab 2007;292: E629-36.

6. Suryawan A, Nguyen HV, Bush JA, Davis TA. Developmental changes in the feeding-induced activation of the insulin-signaling pathway in neonatal pigs. Am J Physiol Endocrinol Metab 2001;281:E908-15.

7. Orellana RA, Suryawan A, Wilson FA, et al. Development aggravates the severity of skeletal muscle catabolism induced by endotoxemia in neonatal pigs. Am J Physiol Regul Integr Comp Physiol 2012;302:R682-90.

8. Barazzoni R, Short KR, Asmann Y, Coenen-Schimke JM, Robinson MM, Nair KS. Insulin fails to enhance mTOR phosphorylation, mitochondrial protein synthesis, and ATP production in human skeletal muscle without amino acid replacement. Am J Physiol Endocrinol Metab 2012;303: E1117-25.

9. Yan L, Lamb RF. Amino acid sensing and regulation of mTORC1. Semin Cell Dev Biol 2012;23:621-5.

10. Davis TA, Fiorotto ML, Burrin DG, et al. Stimulation of protein synthesis by both insulin and amino acids is unique to skeletal muscle in neonatal pigs. Am J Physiol Endocrinol Metab 2002;282:E880-90.

11. O'Connor PM, Bush JA, Suryawan A, Nguyen HV, Davis TA. Insulin and amino acids independently stimulate skeletal muscle protein synthesis in neonatal pigs. Am J Physiol Endocrinol Metab 2003;284:E110-9.

12. Crossland H, Constantin-Teodosiu D, Gardiner SM, Constantin D, Greenhaff PL. A potential role for Akt/FOXO signalling in both protein loss and the impairment of muscle carbohydrate oxidation during sepsis in rodent skeletal muscle. J Physiol 2008;586:5589-600.

13. Stitt TN, Drujan D, Clarke BA, et al. The IGF-1/PI3K/Akt pathway prevents expression of muscle atrophy-induced ubiquitin ligases by inhibiting FOXO transcription factors. Mol Cell 2004;14:395-403.

14. Krawiec BJ, Nystrom GJ, Frost RA, Jefferson LS, Lang CH. AMP-activated protein kinase agonists increase mRNA content of the muscle-specific ubiquitin ligases MAFbx and MuRF1 in C2C12 cells. Am J Physiol Endocrinol Metab 2007;292:E1555-67.

15. Cai D, Frantz JD, Tawa NE Jr, et al. IKKbeta/NF-kappaB activation causes severe muscle wasting in mice. Cell 2004;119:285-98.

16. Cantó C, Gerhart-Hines Z, Feige JN, et al. AMPK regulates energy expenditure by modulating NAD+ metabolism and SIRT1 activity. Nature 2009;458:1056-60.

17. Gerhart-Hines Z, Rodgers JT, Bare O, et al. Metabolic control of muscle mitochondrial function and fatty acid oxidation through SIRT1/PGC1alpha. EMBO J 2007;26:1913-23.

18. Singleton KD, Wischmeyer PE. Distance of cecum ligated influences mortality, tumor necrosis factor-alpha and interleukin-6 expression following cecal ligation and puncture in the rat. Eur Surg Res 2003;35: 486-91.

19. Rathmacher JA, Nissen SL. Development and application of a compartmental model of 3-methylhistidine metabolism in humans and domestic animals. Adv Exp Med Biol 1998;445:303-24.

20. Riché F, Gayat E, Collet C, et al. Local and systemic innate immune response to secondary human peritonitis. Crit Care 2013;17:R201.

21. Yilmaz TU, Kerem M, Demirtaş CY, et al. Increased resistin levels in intraabdominal sepsis: correlation with proinflammatory cytokines and Acute Physiology and Chronic Health Evaluation (APACHE) II scores. Sultan Qaboos Univ Med J 2014;14:e506-12. 


\section{Articles | Manjarín et al.}

22. Spasojević I, Obradović B, Spasić S. Bench-to-bedside review: neonatal sepsis-redox processes in pathogenesis. Crit Care 2012;16:221.

23. Di Domenico A, Hofer A, Tundo F, Wenz T. Mitochondrial protein acetylation mediates nutrient sensing of mitochondrial protein synthesis and mitonuclear protein balance. IUBMB Life 2014;66:793-802.

24. Johnson MA, Vidoni S, Durigon R, et al. Amino acid starvation has opposite effects on mitochondrial and cytosolic protein synthesis. PLoS One 2014;9:e93597.

25. Peterson JM, Bakkar N, Guttridge DC. NF- $\mathrm{KB}$ signaling in skeletal muscle health and disease. Curr Top Dev Biol 2011;96:85-119.

26. Kovacic S, Soltys CL, Barr AJ, Shiojima I, Walsh K, Dyck JR. Akt activity negatively regulates phosphorylation of AMP-activated protein kinase in the heart. J Biol Chem 2003;278:39422-7.

27. Jäger S, Handschin C, St-Pierre J, Spiegelman BM. AMP-activated protein kinase (AMPK) action in skeletal muscle via direct phosphorylation of PGC-1alpha. Proc Natl Acad Sci USA 2007;104: 12017-22.

28. Jørgensen SB, Wojtaszewski JF, Viollet B, et al. Effects of alpha-AMPK knockout on exercise-induced gene activation in mouse skeletal muscle. FASEB J 2005;19:1146-8.

29. Ljubicic V, Burt M, Lunde JA, Jasmin BJ. Resveratrol induces expression of the slow, oxidative phenotype in $\mathrm{mdx}$ mouse muscle together with enhanced activity of the SIRT1-PGC-1 $\alpha$ axis. Am J Physiol Cell Physiol 2014;307:C66-82.

30. Rooyackers OE, Adey DB, Ades PA, Nair KS. Effect of age on in vivo rates of mitochondrial protein synthesis in human skeletal muscle. Proc Natl Acad Sci USA 1996;93:15364-9.

31. Livak KJ, Schmittgen TD. Analysis of relative gene expression data using real-time quantitative PCR and the 2(-Delta Delta C(T)) Method. Methods 2001;25:402-8. 